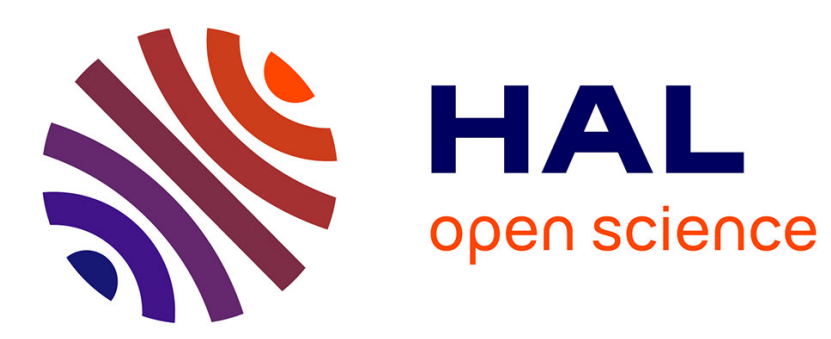

\title{
Reconfigurable Nonlinear Circuit for Wireless Power Harvesting and Backscattering
}

Xiaoqiang Gu, Simon Hemour, Ke Wu

\section{To cite this version:}

Xiaoqiang Gu, Simon Hemour, Ke Wu. Reconfigurable Nonlinear Circuit for Wireless Power Harvesting and Backscattering. 2019 49th European Microwave Conference (EuMC), Oct 2019, Paris, France. pp.543-546, 10.23919/EuMC.2019.8910903 . hal-02527457

\section{HAL Id: hal-02527457 \\ https://hal.science/hal-02527457}

Submitted on 1 Apr 2020

HAL is a multi-disciplinary open access archive for the deposit and dissemination of scientific research documents, whether they are published or not. The documents may come from teaching and research institutions in France or abroad, or from public or private research centers.
L'archive ouverte pluridisciplinaire HAL, est destinée au dépôt et à la diffusion de documents scientifiques de niveau recherche, publiés ou non, émanant des établissements d'enseignement et de recherche français ou étrangers, des laboratoires publics ou privés. 


\title{
Reconfigurable Nonlinear Circuit for Wireless Power Harvesting and Backscattering
}

\author{
Xiaoqiang $\mathrm{Gu}^{\# 1}$, Simon Hemour ${ }^{* 2}, \mathrm{Ke} \mathrm{Wu}^{\# 3}$ \\ \#Poly-GRAMES Research Center, Polytechnique Montreal, Canada \\ ${ }^{*}$ CNRS and IMS Research Center, University of Bordeaux, France \\ $\left\{{ }^{1}\right.$ xiaoqiang.gu, ${ }^{3}$ ke.wu\}@polymtl.ca, ${ }^{2}$ simon.hemour@ims-bordeaux.fr
}

\begin{abstract}
Scarcity of accessible energy compels battery-less micro-energy wireless devices to embed energy harvesting, backscattering modulation, and even harmonic operation to avoid self-jamming. However, since those Internet of Things (IoT) devices are meant to be ubiquitous, keeping a low level of complexity of nonlinear operation, and also a small footprint circuit is becoming an ever-challenging task. This paper presents a hybrid IoT frontend architecture capable of rectification, second-harmonic generation and modulation using a single diode controlled by harmonic terminations. The proposed solutions of re-configurability are validated by measurements.

Keywords - backscattering, energy harvester, harmonic transponder, Internet of Things (IoT)
\end{abstract}

\section{INTRODUCTION}

A large-scale integration of ubiquitously connected and well-rounded low-power sensor/communication platforms is the foundation of Internet of Things (IoT) applications [1]. It is projected that the number of IoT connected devices worldwide will be reaching 75.44 billion in the year of 2025 [2]. As the footprints of such sensor/communication platforms are continuously becoming smaller, powering them turns out a challenging problem. Thus, higher-energy-density batteries are always a first choice at this moment for these distributed platforms for an extended life span [3]. However, considering battery recharging or replacement issues at a large scale, an ultimate long-lasting solution is to develop and deploy selfsustainable sensor/communication platforms [4]. Making a full use of ambient radiofrequency (RF) power to create selfsustainability is a straightforward idea, since this pervasive energy form is actually wasted if not used immediately [5]. Currently, there exist two different ways to recycle the ambient RF power as described in Fig. 1. Converting ambient RF power into dc energy which replaces conventional batteries in the platforms is the first one. The other is to modulate an injecting ambient RF power signal and deliver the modulated signal information, thus realizing a backscattering communication.

Harvesting ambient RF power was proposed decades ago and has now been going through a rapid development [6]. Due to a low energy density, the ambient RF power harvesting still has its power conversion efficiency below $10 \%[7,8]$. With this issue in mind, researchers around the world have been developing techniques and taking measures from multiple aspects. For example, advanced semiconductor process has been continuously rolled out to enhance device nonlinearity [9, $10]$.

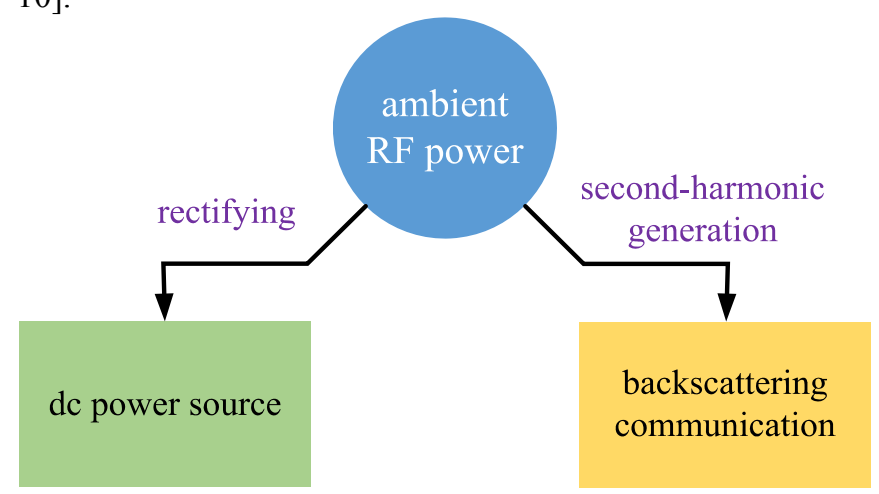

Fig. 1. Two ways to create self-sustainability of sensor/communication platforms based on ambient RF power.

Eliminating matching network to reduce any inherent mismatch loss can boost the power conversion efficiency [11]. As for the technique of backscattering communication including sensing functions, it is an another promising technique, which requires no specific transmitter $[12,13]$. The use of a second-harmonic generation to avoid radar clutter interference and self-jamming has been proved an efficient backscattering communication scheme [14]. In this work, a reconfigurable nonlinear circuit-based frontend is proposed and studied with a reduced system complexity and small form factor to meet the requirements of IoT applications. It combines both harmonic transponder and power harvester but still remains a multifunction compact design, featuring the use of only one diode for both functionalities. The harmonic transponder and power harvester share most of the circuit layout to minimize the circuit size. In this work, preliminary circuit design details are revealed. Also, measurement results related to the circuit performance of this multifunction scheme are provided and discussed in the paper, showing its potential to implement reconfigurable IoT tasks. 


\section{Circuit ARChITECTURE AND DEMONSTRATION}

\section{A. Diode Role}

Diode is the core component of the proposed circuit to produce dc and higher-ordre harmonics. For example, the relationship of current $I$ and voltage $V$ of a Schottky diode can be expressed by:

$$
I_{\text {diode }}=I_{S} \cdot\left[e^{\frac{V_{o n}}{n \cdot V_{t}}}-1\right]
$$

where $I_{s}, n$, and $V_{t}$ are saturation current, ideality factor and thermal voltage of the diode, respectively. $V_{\text {on }}$ is the voltage across diode junction. This $I-V$ relationship can be expanded by Taylor series around the zero bias point [15]:

$$
I_{\text {diode }}=I_{S} \cdot\left[\frac{V_{o n}}{n \cdot V t}+\frac{V_{o n}^{2}}{(n \cdot V t)^{2} 2 !}+\cdots+\frac{V_{o n}^{k}}{(n \cdot V t)^{k} k !}+\cdots\right]
$$

Let us assume that injecting power is a single-tone signal. Thus, $V_{\text {on }}$ can be expressed as $V_{f_{0}} \cos \left(\omega_{0} t\right)$, where $V_{f_{0}}$ is the voltage magnitude and $\omega_{0}$ is the angular frequency. For a lowpower injecting case, the first two terms in (2) can be used as a good approximation for simplicity. Then, by substituting $V_{o n}$ expression into (2), we have:

$$
I_{\text {diode }}=\frac{I_{s} \cdot V_{f_{0}}^{2}}{4(n \cdot V t)^{2}}+\frac{I_{s} \cdot V_{f_{0}}}{n \cdot V t} \cos \left(\omega_{0} t\right)+\frac{I_{s} \cdot V_{f_{0}}^{2}}{4(n \cdot V t)^{2}} \cos \left(2 \omega_{0} t\right)
$$

First and third terms in (3) represent the dc and secondharmonic output in connection with the diode frequency conversion. There, the diode can realize both functionalities of power harvester and harmonic transponder utilizing the secondharmonic generation by selecting a desired output power as shown in Fig. 2. To maximize the diode operation efficiency and at the same time to make a full use of the RF power, only one functionality works at one time.

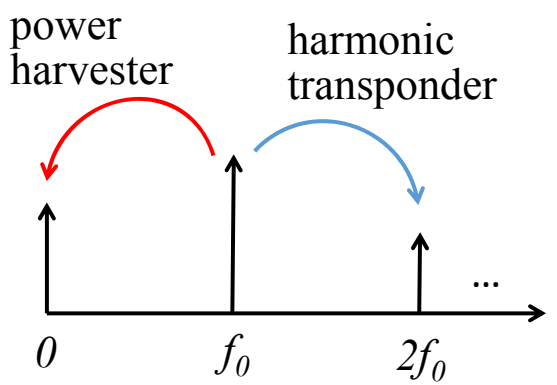

Fig. 2. Selecting desired nonlinear components generated by diode for different functionalities.

\section{B. Harmonic Transponder}

As mentioned earlier, the proposed harmonic transponder using the second-harmonic signal features a high robustness to radar interference clutter [14]. To fully comply with ISM band rules for its operation, the fundamental frequency (uplink) in this work is set at $910 \mathrm{MHz}$, and thus the second-harmonic frequency (downlink) becomes $1820 \mathrm{MHz}$. Fig. 3 presents a schematic of a typical harmonic transponder layout. Besides the input and output matching networks, a set of quarter-wave open-circuited and short-circuited stubs are introduced to enhance the diode frequency conversion from $f_{0}$ to $2 f_{0}[14$, $16]$.

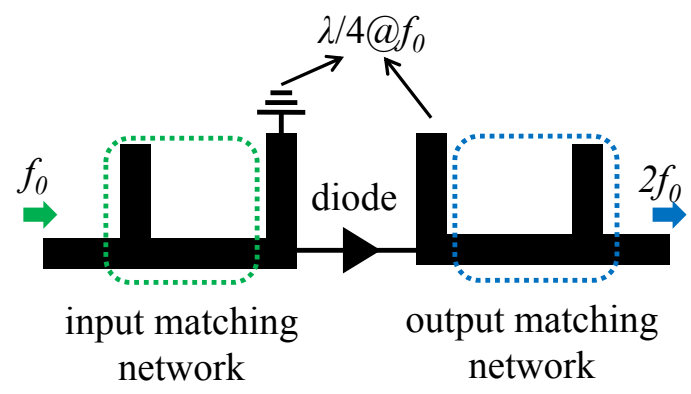

Fig. 3. Schematic of the proposed harmonic transponder using the secondharmonic generation.

\section{Rectifier}

To make a full use of the circuit layout of the harmonic transponder and also to achieve a compact design, a rectifier circuit of interest evolves based on the schematic of the harmonic transponder as shown in Fig. 4. As the core idea devised in this work, only one diode suitable for both functionalities is considered, which is the diode SMS7630 from Skyworks in this demonstration. Its key SPICE parameters are listed below in Table I. This diode has been widely used in lowpower rectifier design [17]. Also, it has been reported that SMS7630 is a suitable candidate for a fully passive harmonic transponder design to reduce conversion loss [14].

TABLE I

KEY SPICE PARAMETERS

\begin{tabular}{|c|c|}
\hline Parameters & SMS7630-079LF \\
\hline$C_{j 0}(\mathrm{pF})$ & 0.14 \\
\hline$I_{s}(\mathrm{~A})$ & $5 \mathrm{e}-6$ \\
\hline$R_{s}(\Omega)$ & 20 \\
\hline$n$ & 1.05 \\
\hline$V_{j}(\mathrm{~V})$ & 0.34 \\
\hline$M$ & 0.4 \\
\hline$C_{p}(\mathrm{pF})$ & 0.16 \\
\hline$L_{p}(\mathrm{nH})$ & 0.7 \\
\hline
\end{tabular}

In this work, the rectifier loading part that includes a load resistor and a filtering capacitor, is placed at the end of the output matching stub. An RF switch will be considered to control the reconfigurable circuit. Other forms of ambient energy such as solar or thermal energy can be introduced to enable the operation of the RF switch in a more mature design afterwards. Once the switch is connected, a dc path is created in the circuit as indicated in Fig. 4 and a power harvesting starts at this moment. When the switch is disconnected, the circuit then 
becomes a harmonic transponder which can be represented by Fig. 3.

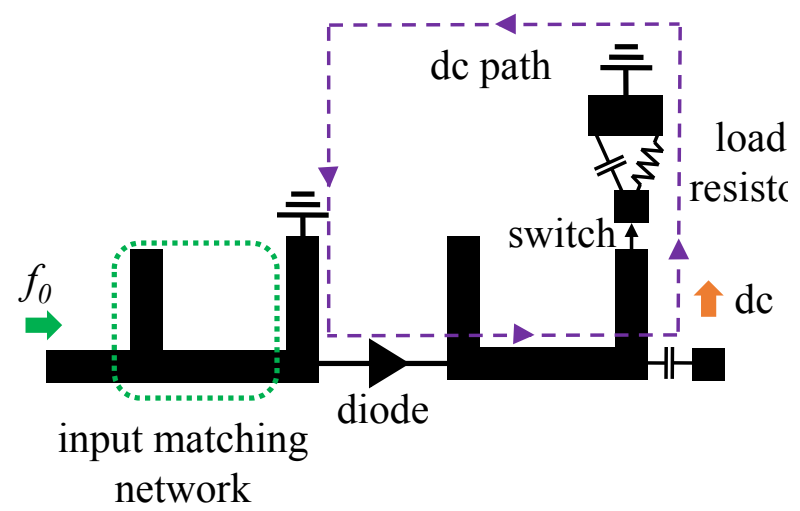

Fig. 4. The proposed circuit working as rectifier when switch is connected with dc path.

The proposed design is first verified by our ADS schematic simulation. Rogers RT/duroid 6010 with a thickness of 10 mils is selected as the substrate for prototyping. Simulation results are presented in Fig. 5. As expected, both functionalities work properly. For example, the conversion loss at an input power of $-30 \mathrm{dBm}$ is $19.7 \mathrm{~dB}$. Rectifying efficiency under the same input level is $8.5 \%$.

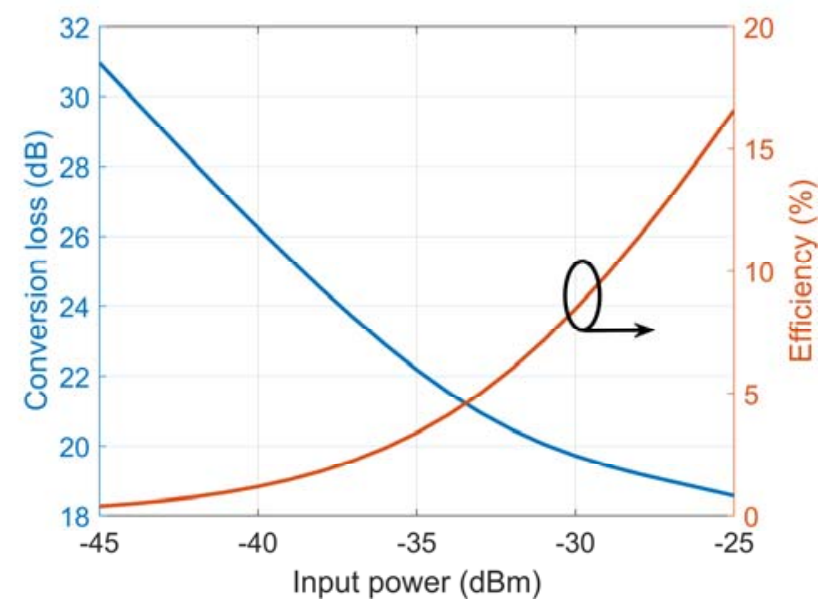

Fig. 5. Performance evaluation of the proposed circuit working as harmonic transponder and energy harvester. Simulation results of conversion loss (left side) and rectifying efficiency (right side).

\section{MEASUREMENT RESUlTS AND DisCUSSIONS}

Fig. 6 shows the experimental prototype of the proposed circuit, whose size is about $73 \mathrm{~mm} \times 45 \mathrm{~mm}$. Major components of the circuit including the diode, harvester load and capacitor located at the output port of the harmonic transponder are labelled. The circuit layout enabling power harvester and harmonic transponder is also highlighted. It can clearly be seen that the circuit layout is efficiently shared by the two different functionalities and the common area accounts for more than 90

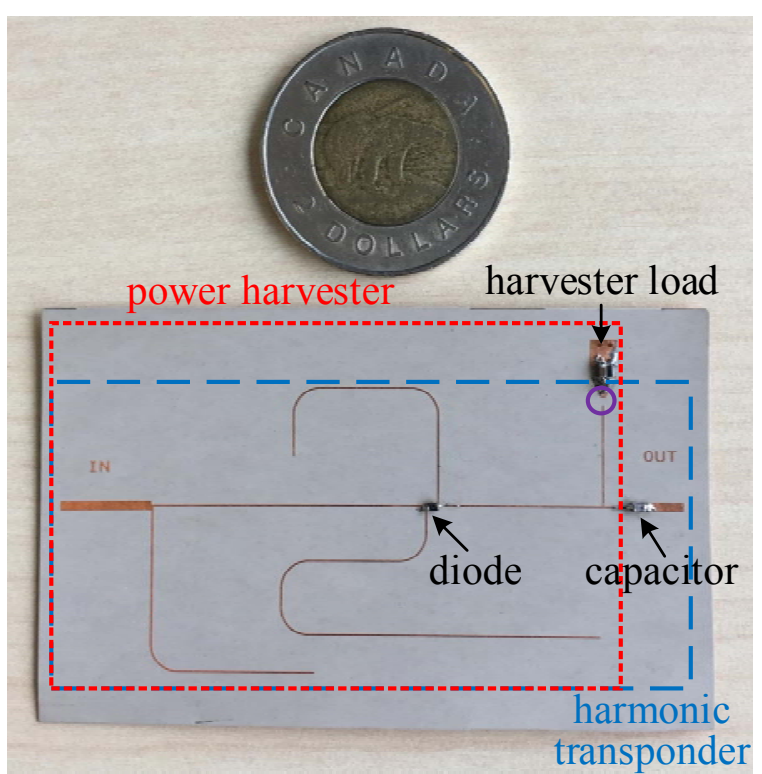

Fig. 6. Photo of the proposed nonlinear circuit prototype. The place reserved for an RF switch is labeled by a circle.

$\%$. Also, we note that the RF switch is not included at this stage, and a $0 \Omega$ resistor is used to mimic the situation when the RF switch is connected.

Performances of the harmonic transponder and power harvester are tested separately with results as shown in Fig. 7. In this design, microstrip lines with a characteristic impedance of $100 \Omega$ is adopted, leading to a narrow linewidth. It may introduce more fabrication errors and transmission losses and thus the matching network performance does not work as well as expected. Finally, as we can see from Fig. 7, measured conversion loss results are slightly higher than simulated counterparts. Meanwhile, harvesting efficiency results are lower compared with simulation ones. However, both conversion loss and harvesting efficiency curves agree with simulation results in a very reasonable manner. Moreover, these

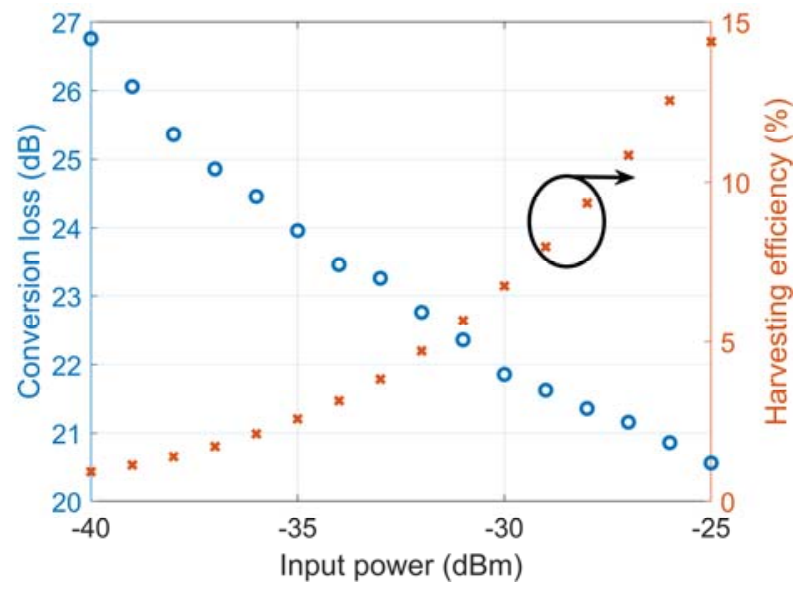

Fig. 7. Measurement results of the proposed circuit working as harmonic transponder and energy harvester. Conversion loss (left side) and harvesting efficiency (right side). 
measurement results indicate that this nonlinear circuit utilizing only one diode can work properly as both harmonic transponder and power harvester. It is believed that through the introduction of an RF switch, this reconfigurable circuit would present a promising design for future IoT applications.

\section{CONCLUSION}

This work proposes and demonstrates a reconfigurable nonlinear circuit for wireless power harvesting and harmonic transponder-based backscattering. This nonlinear circuit features the use of only one Schottky diode to realize both functionalities. More than $90 \%$ of the circuit layout is shared by the passive harmonic transponder and rectifier, thereby showing a super compact design. Preliminary measurement results in this work confirm and demonstrate that this circuit can realize both functionalities of harmonic transponder and rectifier. It is anticipated that an RF switch can then be introduced to make this circuit switchable between both functionalities for future IoT applications.

\section{ACKNOWLEDGEMENT}

The authors would like to express their gratitude to the technical support team of Poly-GRAMES for the excellent fabrication work and measurement support.

\section{REFERENCE}

M. M. Rana and W. Xiang, "IoT Communications Network for Wireless Power Transfer System State Estimation and Stabilization," IEEE Internet of Things Journal, vol. 5, pp. 41424150, 2018.

[2] D. Lund, C. MacGillivray, V. Turner, and M. Morales, "Worldwide and regional internet of things (iot) 2014-2020 forecast: A virtuous circle of proven value and demand," International Data Corporation (IDC), Tech. Rep, vol. 1, 2014.

[3] X. Shen, H. Liu, X.-B. Cheng, C. Yan, and J.-Q. Huang, "Beyond lithium ion batteries: higher energy density battery systems based on lithium metal anodes," Energy Storage Materials, vol. 12, pp. 161$175,2018$.

[4] A. Abdelraheem, M. Sinanis, S. Hameedi, M. Abdelfattah, and D. Peroulis, "A Flexible Virtual Battery: A Wearable Wireless Energy Harvester," IEEE Microwave Magazine, vol. 20, pp. 62-69, 2019.

[5] S. Hemour and K. Wu, "Radio-frequency rectifier for electromagnetic energy harvesting: Development path and future outlook," Proceedings of the IEEE, vol. 102, pp. 1667-1691, 2014.

[6] S. Hemour, X. Gu, K. Wu, and N. Shinohara, "Efficiency of Rectenna," in Recent Wireless Power Transfer Technologies via Radio Waves, ed: River Publishers, 2018, pp. 95-132.

[7] S. Hemour, Y. Zhao, C. H. P. Lorenz, D. Houssameddine, Y. Gui, C.-M. Hu, et al., "Towards low-power high-efficiency RF and microwave energy harvesting," IEEE transactions on microwave theory and techniques, vol. 62, pp. 965-976, 2014.

[8] X. Gu, S. Hemour, L. Guo, and K. Wu, "Integrated Cooperative Ambient Power Harvester Collecting Ubiquitous Radio Frequency and Kinetic Energy," IEEE Transactions on Microwave Theory and Techniques, vol. 66, pp. 4178-4190, 2018.

[9] C. H. P. Lorenz, S. Hemour, W. Li, Y. Xie, J. Gauthier, P. Fay, et al., "Breaking the efficiency barrier for ambient microwave power harvesting with heterojunction backward tunnel diodes," IEEE Transactions on Microwave Theory and Techniques, vol. 63, pp. 4544-4555, 2015.
[10] X. Zhang, J. Grajal, X. Wang, U. Radhakrishna, Y. Zhang, J. Kong, et al., "MoS 2 Phase-junction-based Schottky Diodes for RF Electronics," in 2018 IEEE/MTT-S International Microwave Symposium-IMS, 2018, pp. 345-347.

[11] C. Song, Y. Huang, J. Zhou, P. Carter, S. Yuan, Q. Xu, et al., "Matching network elimination in broadband rectennas for highefficiency wireless power transfer and energy harvesting," IEEE Transactions on Industrial Electronics, vol. 64, pp. 3950-3961, 2017.

[12] V. Liu, A. Parks, V. Talla, S. Gollakota, D. Wetherall, and J. R. Smith, "Ambient backscatter: wireless communication out of thin air," in ACM SIGCOMM Computer Communication Review, 2013, pp. $39-50$.

[13] F. Amato and S. Hemour, "The Harmonic Tunneling Tag: a DualBand Approach to Backscattering Communications," in 2019 IEEE International Conference on RFID, 2019, pp. 1-3.

[14] X. Gu, S. N. Nallandhigal, L. Guo, S. Hemour, and K. Wu, "Diplexer-Based Fully Passive Harmonic Transponder for Sub-6 GHz 5G-Compatible IoT Applications," IEEE transactions on microwave theory and techniques 2018.

[15] C. H. Lorenz, S. Hemour, W. Liu, A. Badel, F. Formosa, and K. Wu, "Hybrid power harvesting for increased power conversion efficiency," Microwave and Wireless Components Letters, IEEE, vol. 25, pp. 687-689, 2015.

[16] X. Gu, L. Guo, S. Hemour, and K. Wu, "Analysis and Exploitation of Diplexer-based Fully Passive Harmonic Transponder for 5G Applications," presented at the 2018 IEEE MTT-S International Microwave Workshop Series on 5G Hardware and System Technologies (IMWS-5G 2018), Dublin, Ireland, 2018.

[17] D. Bui, T. Vuong, J. Verdier, B. Allard, and P. Benech, "Design and Measurement of 3D Flexible Antenna Diversity for Ambient RF Energy Scavenging in Indoor Scenarios," IEEE Access, 2019. 